\title{
A BLOCKCHAIN-BASED APPROACH TO ENABLE REMOTE SENSING TRUSTED DATA
}

\author{
Miguel Pincheira ${ }^{1,2}$, Elena Donini $^{1,2}$, Raffaele Giaffreda $^{1}$, Massimo Vecchio $^{1}$ \\ ${ }^{1}$ Fondazione Bruno Kessler, Trento, Italy \\ ${ }^{2}$ Department of Information Engineering and Computer Science, University of Trento, Trento, Italy
}

KEY WORDS: Open Data, Blockchain, Precision Agriculture, Remote Sensing

\begin{abstract}
:
Remote sensing considerably benefits from the fusion of open data from different sources, including far-range sensors mounted on satellites and short-range sensors on drones or Internet of Things devices. Open data is an emerging philosophy attracting an increasing number of data owners willing to share. However, most of the data owners are unknown and thus, untrustable, which makes shared data likely unreliable and possibly compromising associated outcomes. Currently, there exist tools that distribute open data, acting as intermediaries connecting data owners and users. However, these tools are managed by central authorities that set rules for data ownership, access, and integrity, limiting data owners and users. Therefore, a need emerges for a decentralized system to share and retrieve data without intermediaries limiting participants. Here, we propose a blockchain-based system to share and retrieve data without the need for a central authority. The proposed architecture (i) allows sharing data, (ii) maintains the data history (origin and updates), and (iii) allows retrieving and evaluating the data adding trustworthiness. To this end, the blockchain network enables the direct connection of data owners and users. Furthermore, blockchain automatically interacts with participants and keeps a transparent record of their actions. Hence, blockchain provides a decentralized database that enables trust among the participants without a central authority. We analyzed the potentials and critical issues of the architecture in a remote sensing use case of precision farming. The analysis shows that participants benefit from the properties of the blockchain in providing trusted data for remote sensing applications.
\end{abstract}

\section{INTRODUCTION}

Recently, space agency policies on open data access encourage the development of various automatic methods to extract useful information for a wide range of Earth and Planetary science and applications. For instance, the European Space Agency provides Sentinel-2 data with a globe coverage and a high revisit frequency at no cost to the public though the Copernicus Programme. The spatial resolution (10 to $60 \mathrm{~m}$ ) of these images is relatively high, but not enough for all applications. Hence, data fusion methods consider data from different short-range sources as complementary of remote sensing sensors.

The philosophy of open data becomes more relevant with the enormous quantity of data collected nowadays by close-range sensors, e.g., personal drones, IoT sensor networks, and open government data. Even if close and far range sensors acquire data on the same scene, they focus on different properties. These two types of data are complementary, and remote sensing (RS) can take advantage of close-range data and integrate them to generate more consistent, accurate, and useful products. For instance, close-range data as IoT measurements have a higher spatial and temporal resolution than ESA's Sentinel-2, but limited coverage. On the contrary, far-range data provide full coverage of the terrestrial surface with a relatively low spatial resolution. Moreover, close-range data available from in-situ (e.g., IoT measurements) are of particular importance for the validation of RS methods and products. However, owners of shortrange data are part of a heterogeneous group, e.g., research institutions or startup owners, and are often unknown. Therefore, the owners and the data are not fully trustable, and thus, using the data may compromise the associated research outcome.

Currently, sharing and retrieving close-range data is possible through some tools mostly managed by intermediaries, e.g., open data portals. Intermediaries define the policies for data ownership and access and set the rules to evaluate data reliability and integrity. These tools tend to favor usability over transparency since they aim at connecting data owners with users. Therefore, a need emerges for a system that enables sharing and retrieving data without a limiting central authority.

This paper proposes an architecture based on the blockchain to build a network to share and validate data acquired by untrusted sources. Blockchain acts as an intermediary connecting data owners and users- its is a decentralized database of trusted data accessible to all the participants. The intrinsic properties of blockchain enable trust and remove the necessity to have a third party validating the interactions among the participants. To investigate the proposed architecture, we analyze a use case to identify the critical issues and the potentialities of the system. We consider precision agriculture as it is well known for combining different types of data, e.g., IoT measurements and satellite optical images, to monitor the status of crop fields.

\subsection{Related Work}

Recently, the interest in blockchain increased since users can monetize their resources and profit from them without an intermediary. Energy trading (Mengelkamp et al., 2018) and IoT data marketplaces (Ramachandran et al., 2018) are just two examples where blockchains enable direct interactions between participants without a validating intermediary. Moreover, the auditability property allows the identification of the owner of each resource and the direct incentivizing of the participants contributions.

In remote sensing, only a few works explore the benefits of blockchain. In a whitepaper, ESA highlighted the importance of integrating blockchain in remote sensing applications 
(European Space Agency, April 2019). On the one hand, blockchain enables the transfer of value- it is a public network where to share and retrieve data without a central authority. Moreover, smart contracts allow automating the actions, such as elaborating information. On the other hand, short-range sensors acquire everyday a large amount of data useful for Earth Observation. However, data owners lack a tool where to share data while keeping ownership. Further, the data cannot be trusted since data owners are unknown and thus, not reliable. Blockchain is the solution to build a system to generate trust among users without an intermediary.

Blockchain is a decentralized database that keeps track of the flow of information. Blockchain technology is verifiable and immutable by default- all the actors can access the information and its changes over time. For this reason, blockchain is suggested as a distributed database to share the knowledge on land ownership (Ellehauge, 2017) (Kempe, 2017), geohashing (Ellehauge, 2017), i.e., to create a geodesic grid of the world (Ellehauge, 2017), and to share geodata in an open way, e.g., public map, without relying on a central authority, e.g., Google Maps or OpenStreetMap.

In the literature, there exist few works exploiting blockchain to store and share data in a trustworthy and auditable manner. (Molesky et al., 2018) propose a blockchain as a database to track satellites and debris orbits. The database has two configurations- history data configuration and sliding window configuration. In the first, all the information is available to all the network peers. In the second, only the information on the last 48 hours is available to all the network, while selected peers stores all history. Here, blockchain technology preserves data integrity and provides smart contract to automatize some actions on the data. However, the system does not exploit other advantages of the technology, e.g., trust and incentives, as all the actors are already known. In (Lin et al., 2017), a blockchain database stores the national data on water quality acquired by an irrigation system spread in the Taiwan area. The data benefit of the blockchain characteristics, i.e., decentralization, immutability, and auditability, however, trust is not a necessary since all the data sources are known. Moreover, the system stores only data from IoT sensors, which is far from the purpose of this paper. Finally, (Leka et al., 2019) proposes a blockchain system to store and share geospatial data among users, particularly researchers. The system uses blockchain and smart contracts to develop a complex reward mechanism to engage the participants. However, the data are in global storage, which eliminates the decentralization and introduces a controlling authority that decide the rules to access the data.

The rest paper is organized as follows-Section 2 illustrates the fundamentals on the blockchain technology. Section 3 proposes architecture with the details on the actors, the interactions, and the smart contracts. In Section 4, we analyze the proposed architecture with a use case on precision agriculture to identify its potentialities and issue. Finally, Section 5 concludes the paper and presents future works.

\section{GENERAL CONCEPTS ON BLOCKCHAIN TECHNOLOGY}

Blockchain is a recent and promising technology still at an early stage of research and development, however it offers a unique combination of features, arising the interest in several fields. Here, we want to use a blockchain-based system to enable the trust in sharing and retrieving data from unknown sources for RS purposes without an intermediary 1 . This Section describes the blockchain technology and the features exploited in the propose architecture.

Blockchain combines data structures, incentive mechanisms, and cryptography techniques to maintain a distributed database on a peer-to-peer network. This database stores the information by using logical blocks linked to each other and thus creating a chained data structure. The first blockchain use case stored financial transactions between unknown parties, i.e., cryptocurrency. However, the technology has the potential to be adopted in any other use cases where the data exchange occurs between untrusted parties. Hence, blockchain enables trust without an intermediary by providing a transparent and immutable list of records.

The three main components of a blockchain system are the transactions, the blocks, and the state of the network. Transactions represent the information transfer among two peers that are grouped into the blocks. A block is the data structure that contains the information, i.e., a group of transactions, validated by the peers in the network. The information in the blocks creates a unique global state that is agreed upon by all the network peers.

The blockchain system is managed by a protocol that indicates (i) how to create and validate transactions, (ii) how to create and validate new blocks, and (iii) how to broadcast the block and update the state of the network. Figure 1 shows the step of the protocol during the interaction of two peers. The next subsections explain in more details the steps of the protocol and the main features that enables trust among peers.

Transaction creation and validation. In a blockchain system, each peer has a unique address associated with a pair of cryptographic keys. Peers use these keys (i) to sign their transactions to certify their origin and (ii) to validate the integrity and the origin of the transaction they receive. Blocks collect valid transactions and are distributed in a peer-to-peer network, and thus are acessible to any peer. Hence, blockchain is a transparent record of interactions (property of auditability).

Block creation and validation. A block is a time-stamped data structure that contains a group of transactions and the link to the previous block. When creating a block, the protocol applies cryptographic techniques for its validation and the sealing. Validating the block requires the generation of the nonce, which is a unique pseudo-random number obtained by solving a cryptographic puzzle (consensus protocol). Sealing demands a hashing function that creates a unique identifier (Block ID) based on the block content and nonce. The identifier protects from the tampering since it loses its validity with any change of the data in the block. The link with the previous block creates a retroactive relation that contributes to secure the blockchainany modification implies heavy computations to validate and seal the previous and following blocks. Thus, the information in blockchains is considered permanent in time (property of immutability).

Block broadcasting. When a new block is broadcasted to the network, each peer appends it to the local copy of the chain. Since the blockchain replicates in each peer, the entire system has a distributed architecture, and thus, it is tolerant of data failures. Appending the block implies its validation and the update of the blockchain global state that is agreed by all 


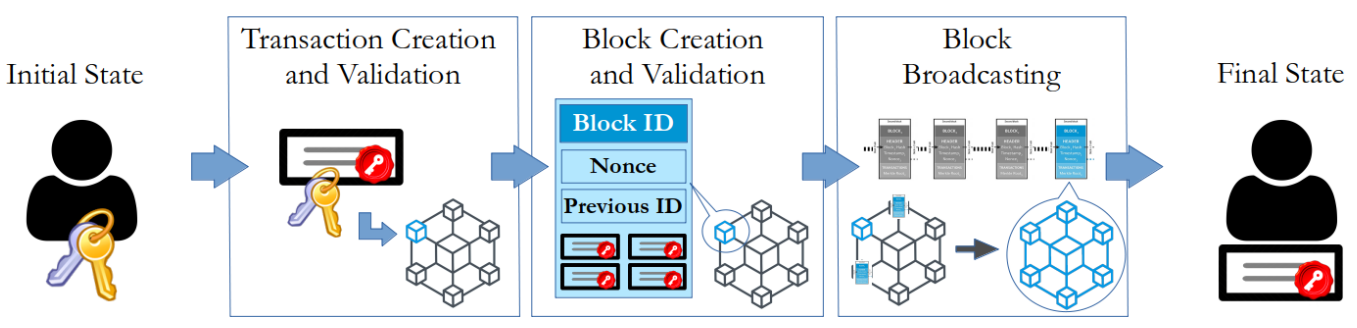

Figure 1. Flowchart of the blockchain protocol.

the peers. To interact, all peers follow a consensus protocol that indicates how to resolve conflicts, avoid abuses related to personal interests over the common good, and incentive participants. In this way, interactions occur directly among peers without any central control. Hence, the consensus protocol is what makes the system decentralized, which is an intrinsic property of blockchain.

\subsection{Benefits of blockchain}

Blockchain has three main embedded properties- auditability, immutability, and decentralization. Auditability indicates that all network peers can validate the integrity and origin of the data using cryptographic techniques. This removes the blind trust in the central authority that characterizes existing tools to share and retrieve data. Immunatibilty implies that blockchain is hardly alterable without using massive computational resources. Hence, actors have full access to the complete history of the data, including the origin and following updates. Decentralization is related to the consensus protocol, which provides a global status of the system, agreed among the peers without a central control. The combination of the three properties enables trust among peers, based on the agreed global state of the network, and removes the need for a validating intermediary.

The central protocol supports additional features that can improve the functionalities of a blockchain system. Ethereum, a blockchain implementation, introduced smart contracts as an agreement between two or more peers. A smart contract is a distributed software running on top of the blockchain network. The software runs deterministically in all the peers at the same time to process the information in the blockchain. The same execution output enforces the agreement among peers without the need for any third-party validator. Thus, smart contracts provide the mean to automatically create and manipulate information in a trustworthy and transparent manner.

\section{PROPOSED ARCHITECTURE}

The paper aims at defining a distributed architecture that collects the information on the data, i.e., the metadata, shared by untrusted data sources. We use a blockchain-based approach to collect, validate, and track the metadata. Blockchain acts as a distributed infrastructure that provides a secure, immutable, and transparent record of the metadata. This Section will describe the architecture with a focus on the actors and the interactions. We first describe the actors, which are the blockchain peers that share and validate the metadata. Then, we analyze the possible interactions between the actors and the blockchain. Finally, we describe the smart contracts that enables the interaction between the actors.

\subsection{Actors}

Data comes from different sources that are trusted, e.g., space agencies and universities, or untrusted, e.g., private companies and volunteers. In this paper, the peers are untrusted since we aim at providing value to the data that otherwise cannot be trusted. We consider as untrusted actors those that voluntarily collect and share data, as Figure 2 shows. Some examples of common actors are- the company sharing data acquired by IoT sensors on the temperature and the humidity of a crop field; the research group sharing a database on multitemporal images acquired by optical and SAR sensors; and finally, the people that shares pictures taken by a drone or a cellphone.

\subsection{Interactions}

Interactions happen between actors and the blockchain-based system. Here, actors can (i) share the metadata of a dataset via a transaction to a smart contract, (ii) use smart contracts to search in the dataset list, and finally (iii) use smart contracts to retrieve the dataset metadata. These interactions are associated with a reward for the actor and a quality evaluation of the dataset as an incentive to use the system. The score indicates the experience that the actor had with the dataset, including how representative the metadata are of the dataset, the quality of the data and of the dataset structure. However, the quality score should be define with criteria specific for the use case. The following paragraphs describe the interactions and the reward and evaluation mechanisms.

Sharing information. In this architecture, the blockchain is a container of information on different datasets. Blockchain maintains a list whose records are the metadata of the dataset shared by actors. The metadata is shared by sending transactions to the smart contract in a standard format. The smart contract verifies the transactions with predefined rules and rewards the actors. Moreover, the contract inserts the metadata in the dataset list-it adds a new record for a new dataset or updates the record for an existing one. Blockchain stores a history of all the events so that changes are traceable and accountable.

Searching for information. Actors looking for a specific dataset can query the smart contract for matching records in the dataset list. The smart contract searches the list with some criteria given by the actor. The contract retrieves the information on the matching datasets and provides it to the actor. Thus, the actor receives the part of the metadata and the current quality score of each the dataset. With this information, the actor can decide which dataset to use and require the complete metadata.

Accessing information. Considering the blockchain as a list of datasets, an actor can ask the smart contract to access the information related to a particular dataset. Retrieving the data implies that the smart contract transmits the complete metadata of that dataset to the actor. With this information, the actor can 


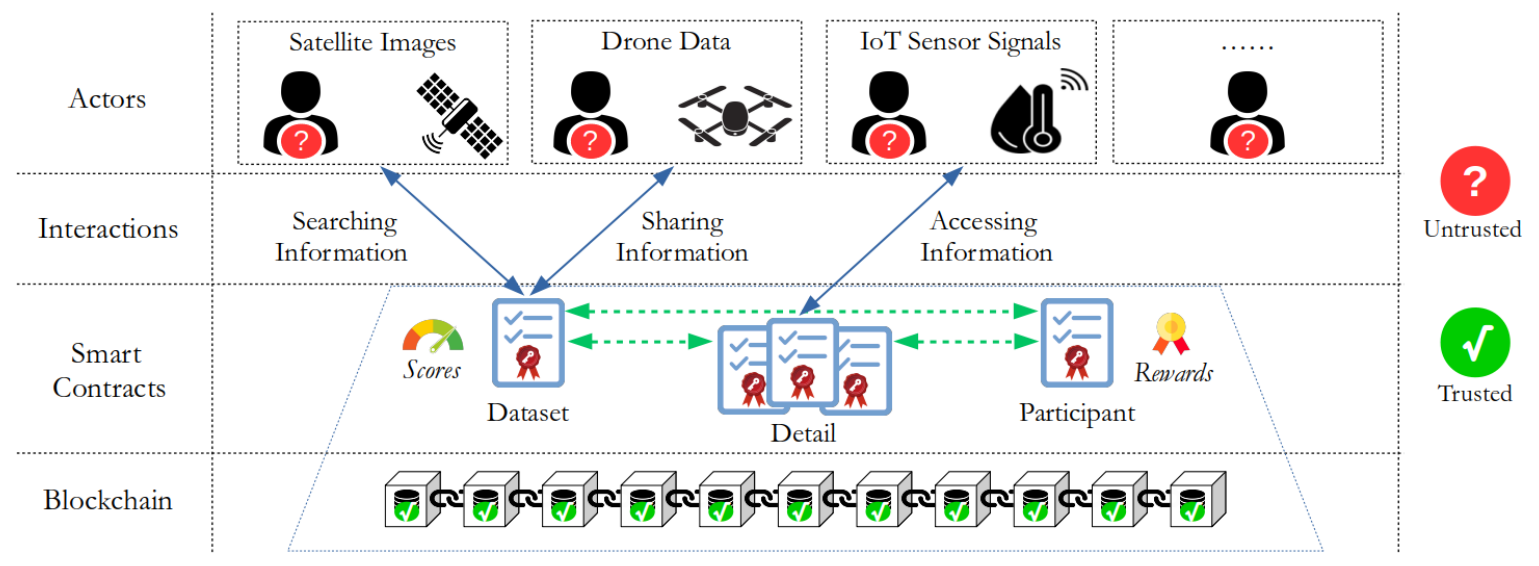

Figure 2. Structure of the proposed architecture.

access and use the data. In exchange for the metadata, the actor is asked to give an evaluation of the quality of the dataset. If the actor provides this evaluation, it receives a positive reward, otherwise a negative one. Based on these rewards, smart contracts can limit the actor to have further access to other datasets.

\subsection{Smart Contracts}

Transactions are the interactions between actors and the blockchain system, i.e., the smart contracts. The smart contracts define rules and methods to validate and process the information in the transactions. They provide an interface to access information, making it available for actors and other smart contracts. Moreover, a relevant characteristic of smart contracts is the ability of creating and adding information in the blockchain. Here, these interactions translate into rewards for the actors and a quality score for the datasets. We employ three types of smart contracts: Participant, Dataset, and Detail.

Participant smart contract. The Participant smart contract manages the rewards of each actor according to predefined rules. The Participant contract receives transactions by the (i) Dataset contract when the latter adds a new record in the dataset list and (ii) Detail contract when an actor uses a dataset. The transaction contains the information on the actor and its rewarding. Hence, the contract manages the list of the actors and the history of their rewards. The list is accessible by actors and other smart contracts at any time, which is central for granting or denying access to datasets.

Detail smart contract. The Dataset contract creates a new Detail contract for each new dataset shared in the network to store metadata. Each Detail contract manages the metadata of a database and accepts the updates coming from the actor who originally shared the data. Each Detail contract is an independent unit of information more accessible than the blocks in the chain. Saving the information in the blockchain grants trust, while the contract provides a simpler way to access the metadata. Moreover, the Detail contract can provide the information in a meaningful way to the actor, e.g., only specific fields of the metadata.

The Detail smart contract implements the methods to (i) update and (ii) access the metadata. (i) Updating the metadata requires a transaction with the changes of the dataset. The contract processes only the transactions coming from the same actor who originally shared the dataset. Detail smart contract keeps track of all the changes, which are accessible by anyone in the system. (ii) Accessing dataset metadata requires a transaction to the Detail contract asking for that particular dataset. The contract first verifies if the actor is allowed to access the data in the Participant contract. Secondly, after sharing the metadata, the contract requests the actor to provide a quality score to the Datasets contract. Evaluating the dataset grants a positive reward and the further use of the system. On the contrary, the actor receives a negative reward when refusing to provide the quality score. Evaluating a dataset is of critical importance for the system- it helps in validating the information shared by the actors and thus checking the quality of the data in the blockchain.

Dataset smart contract. The Dataset smart contract keeps a list of the existing datasets and their quality scores. Moreover, it indicates how to (i) share, (ii) browse, and (iii) score the datasets in the systems. (i) To share an dataset, an actor sends a transaction with the dataset metadata coded in a standard format. First, the contract verifies the format of the metadata, then it adds a new record in the dataset list, and finally, it rewards the actor. When adding a new record, the contracts generate a Detail contract with a unique blockchain address stored in the dataset list. (ii) Browsing a dataset implies that an actor sends a transaction with search criteria. The contract uses the criteria to filter the dataset list and returns the results to the actor. The results contain the identifier of the datasets matching the criteria, part of the related metadata, and the current quality score. (iii) Scoring a dataset is done by the actors analyzing the datasets. Actors are asked to send a transaction indicating the quality evaluation of the data they analyzed.

\subsection{Challenges}

The success of participatory systems strongly depends on the number of participants- here the participants are the actors sharing and using the information in the blockchain network. The more are the actors involved, the more the blockchain will be successful and collect further information. Thus, a crucial element is the definition of an incentive mechanism to attract actors in the system. The main incentive is the possibility to browse in the list of datasets stored in the blockchain and then acquiring the information on accessing the dataset. Nevertheless, we decided to add a rewarding mechanism based on a gaming approach to engage more the actors. Each actor, according to its actions, receives a positive or negative reward that accumulates over time. Here, we propose a set of rules for the rewarding mechanism, but any other can be integrated since the system is modular. Thus, actors are encouraged to participate actively to increase their score and gain reliability. 


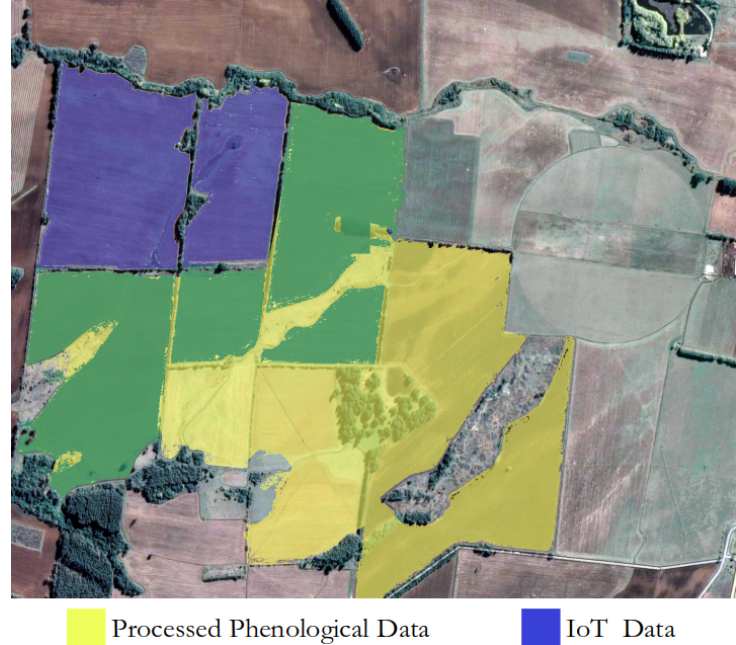

Figure 3. Geographic extension of the datasets shared in the blockchain system- in blue, the IoT raw and processed data, and in yellow, the processed Sentinel-2A data.

Another critical issue is the definition of a standard format for the interactions between actors and smart contracts. The actors must send messages satisfying a shared and previously agreed protocol. The protocol indicates the message structure for all interactions, e.g., the fields required and their order. Having a structured information allows smart contracts to automatize the tasks required by the actor. Smart contracts automatically interact with the actors and elaborate the data in the blockchain. Thus, blockchains can be considered autonomous systems able to self-organize.

\section{USE CASE: PRECISION AGRICULTURE}

This Section analyzes the proposed architecture by describing a use case where actors interact with the blockchain to share and retrieve information to investigate the potentialities and critical issues. We consider a use case in precision farming where the aim is monitoring the phenological cycle of crop fields by fusing different data. As an example, let us assume three actors interacting on the blockchain with different willings and needs. The following paragraphs describe the actors, their needs, and the issues that a blockchain-based system can help to overcome.

\subsection{Actors}

Actor $\mathrm{A}$ is a landowner that aims at developing an automatic system to manage crop fields, in green in Figure 3. He wants to estimate several parameters to monitor the evolution of crop fields and control actuators, such as those for watering and giving fertilizer. Actor A wants to retrieve these parameters, or in the worst-case estimate them, from a reliable list of datasets. Hence, actor A needs an easily accessible system, without a central control setting the rules to limit the actions of the users. Further, the system needs to guarantee the integrity of the data, i.e., the data can not be modified. As an extra feature, the system provides feedback as a quality score assigned by the other users according to their experience.

Actor B is a researcher developing methods for extracting parameters to monitor the phenological cycles of crop fields from Sentile-2A images, in yellow in Figure 3. B wants to share the research outcome while preserving the authorship of the data, and thus the credit. Hence, B needs a sharing system that i) tracks the origin and possible changes, ii) guarantees data integrity, i.e., data cannot be modified, and iii) assures access to everyone. As an additional feature, the system can provide feedback to the data owner based on the scores from the actors that used those data.

Actor $\mathrm{C}$ is an IoT startup that deploys sensors to monitor crop fields, in blue in Figure 3- the startup installed 20 sensors in 1 $\mathrm{km}^{2}$ crop field, which acquire temperature and humidity measurements every 15 minutes. C owns raw data from the sensors and processed data, i.e., the temperature and humidity profile for the past years. $\mathrm{C}$ wants to have a record of the startup achievements and thus share the data in a transparent external system. Sharing the data is a way to present the outcome of the startup to investors and to attract new clients. However, actor $\mathrm{C}$ needs to preserve the ownership of the data, which is challenging with the existing centralized tools. The central authority sets the rules for sharing and retrieving data in the system. Hence, C may face several problems, e.g., with data ownership and data monetization.

The willingness and needs of the actors are diverse and hardly fulfilled by existing systems to share and retrieve data. Existing tools rely on a central authority that acts as an intermediary between untrused actors. The central control sets the rules of the system and thus should be blindly trusted by the actors. The rules indicate ownership and access policies- the former defines the possible change in ownership when uploading the data. The latter shows the accessing mechanism and requirements, which can be restrictive to some actors. Further, the intermediary is the single point of failure of the entire system- any interruption of the services directly compromises the data availability. Finally, actors must trust the central authority on the data integrity since the existing tools usually do not provide enough information to check the validity of the data, i.e., understand if the data are modified. In fact, the central authority has the complete control of the shared data and thus can modify the data or the quality evaluation.

A blockchain-based system overcomes these issues- the decentralized nature of the blockchain eliminates the controlling authority, and thus, the actors can directly interact with the network without the intermediary. Further, since blockchain is a distributed database, there is not a single point of failure, and the data is always accessible to any network peer. Moreover, the blockchain tracks all the interactions with the actors creating a history of the data origin and updates. This information is validated with cryptography techniques and agreed by all the network peers- this guarantees and preserves the data ownership and integrity. Finally, actors provide an evaluation of the data quality, removing any third-party interference as in the case of the existing tools.

A blockchain-based system is highly modular and enables the smooth integration of further services. For example, cryptocurrencies can easily be integrated to monetize the data and incentive participants. The blockchain system facilitates cryptocurrency payments with a marginal cost, which can be done automatically by smart contracts after each transaction. On the contrary, a centralized system does not enable such an effortless way to monetize the data.

\subsection{Interactions}

Sharing information. In our use case, B and $\mathrm{C}$ are the actors willing to share data- they send transactions to the blockchain 
with the information on the datasets. The blockchain validates the transactions with cryptography techniques and stores the data. Hence, blockchain-based systems remove the third-party intermediary that manages the data. Further, since the network peers have a copy of the blockchain, the system lacks a central authority overcoming the issues of existing tools to share and retrieve data.

Searching for information. Here, actor A wants to browse the list of shared datasets- A sends a transaction to the blockchain with filtering criteria. The blockchain system replies with the list of datasets matching the filters. Actor A can trust the integrity of the data since blockchain enables the immutability of the stored data. Moreover, the decentralized architecture of blockchain guarantees these results not to be accessible to everyone, without being censored or promoted.

Accessing information. Actor $\mathrm{A}$ is interested in the raw and processed data previously shared by $\mathrm{B}$ and $\mathrm{C}$. Hence, A requests access to the datasets, by sending a transaction to the blockchain. The blockchain returns access information, which includes the integrity check and access credentials. The metadata accounts for the dataset updates and the change history of the dataset. Hence, the blockchain keeps track of the data owner and the update of the data, e.g., analyses and processing by other actors. Further, the blockchain provides the information to verify the validity of the dataset, i.e., if any modification occurred in the originally shared data.

\section{CONCLUSIONS AND FUTURE WORKS}

The amount of available far-range and close-range data is increasing every day, enabling the development of a large number of remote sensing applications. These applications take advantage of the fusion between close-range data with classical satellite images. Moreover, close-range data, such as those from IoT sensor measurements, can be used as ground truth to validate the result of the automatic methods. However, the owner of close-range data are part of a heterogeneous group and are usually unknown. Thus, using these data may compromise the research outcome. Moreover, existing tools to share and retrieve data have a central authority that acts as an intermediary for the users. However, the presence of the intermediary introduces concerns on data ownership, access, quality, and integrity.

Here, we propose a blockchain-based architecture that (i) enables data owners to share the data without an intermediary, (ii) keeps track of the data updates and provides a quality score, and (iii) overcomes the issue of the untrusted data owners. Any actor can share and retrieve data from the blockchain without caring for the reliability of the data source. Thus, there is no need for a third party that validates the data as the blockchain enables trust among the actors. Smart contracts manage the interactions between actors and the blockchain. For actors, contracts are an interface of the blockchain that simplifies accessing the data. For the blockchain, contracts are entities that elaborate and generate new information. We analyzed the critical issue and the potentialities of the proposed architecture with a remote sensing use case, i.e., precision farming. We conclude that such a system provides benefits to all the actors since sharing and retrieving data do not require the presence of an intermediary.

As future works, we plan to analyze other use cases with a higher number of shared datasets to examine the system scalability and robustness. In that scenario, we plan to implement the system with both public and private blockchains, i.e., Ethereum and Hyperledger Fabric, to evaluate the network performances. Further, we plan to study an innovative storage system to decentralize not only dataset metadata but also the data.

\section{REFERENCES}

Ellehauge, J., 2017. Blockchain in Geospatial Applications. GIM INTERNATIONAL-THE WORLDWIDE MAGAZINE FOR GEOMATICS, 31(5), 43-45.

European Space Agency, April 2019. Blockchain and earth observation. Whitepaper.

Kempe, M., 2017. The Land Registry in the blockchaintestbed. A development project with Lantmäteriet, Landshypotek Bank, SBAB, Telia company, ChromaWay and Kairos Future.

Leka, E., Lamani, L., Selimi, B., Deçolli, E., 2019. Design and implementation of smart contract: A use case for geo-spatial data sharing. 2019 42nd International Convention on Information and Communication Technology, Electronics and Microelectronics (MIPRO), IEEE, 1565-1570.

Lin, Y.-P., Petway, J., Anthony, J., Mukhtar, H., Liao, S.-W., Chou, C.-F., Ho, Y.-F., 2017. Blockchain: The evolutionary next step for ICT e-agriculture. Environments, 4(3), 50.

Mengelkamp, E., Notheisen, B., Beer, C., Dauer, D., Weinhardt, C., 2018. A blockchain-based smart grid: towards sustainable local energy markets. Computer Science-Research and Development, 33(1-2), 207-214.

Molesky, M. J., Cameron, E. A., Jones, J., Esposito, M., Cohen, L., Beauregard, C., 2018. Blockchain network for space object location gathering. 2018 IEEE 9th Annual Information Technology, Electronics and Mobile Communication Conference (IEMCON), 1226-1232.

Ramachandran, G. S., Radhakrishnan, R., Krishnamachari, B., 2018. Towards a decentralized data marketplace for smart cities. 2018 IEEE International Smart Cities Conference (ISC2), 1-8. 